\title{
ON A THEOREM OF ERDÖS AND FUCHS IN ADDITIVE NUMBER THEORY
}

PAUl T. BATEMAN, EUGENE E. KOHLBECKER, AND JACK P. TULL ${ }^{1}$

1. Introduction. Let $a_{1}, a_{2}, \cdots$ be an infinite sequence of integers such that $0 \leqq a_{1} \leqq a_{2} \leqq \cdots$ and $a_{k} \rightarrow+\infty$. If $n$ is a non-negative integer, let $r(n)$ be the number of ordered pairs $(i, j)$ of positive integers such that $a_{i}+a_{j} \leqq n$. Thus if

$$
g(z)=\sum_{k=1}^{\infty} z^{a_{k}}=\sum_{n=0}^{\infty} c_{n} z^{n} \quad(|z|<1),
$$

then

$$
\sum_{n=0}^{\infty} r(n) z^{n}=g(z)^{2} /(1-z) \quad(|z|<1) .
$$

Erdös and Fuchs [1] have shown that if $c>0$, it is impossible to have

$$
r(n)=c n+o\left(n^{1 / 4}(\log n)^{-1 / 2}\right)
$$

as $n \rightarrow+\infty$. In this note we generalize their result to the case where the main term has the form $n L(n)$, where $L$ is a slowly oscillating function which is convex or concave on some interval of the form $[a,+\infty)$.

By a slowly oscillating function we mean a positive-valued continuous function defined on some interval of the form $[a,+\infty)$, where $a>0$, such that $L(c x) / L(x) \rightarrow 1$ as $x \rightarrow+\infty$ for each fixed positive $c$. For the basic information about slowly oscillating functions see [3]. We shall need the fact that if $\epsilon$ is a given positive number, then there exists a positive number $C_{\epsilon}$ such that

$$
L(v x) / L(x)<C_{\epsilon}\left(v^{e}+v^{-\epsilon}\right) \quad(x \geqq a, v x \geqq a) .
$$

The inequality (3) follows readily from the Karamata representation theorem for slowly oscillating functions (cf. [3]).

Our theorem is as follows.

THEOREM. If $L, L_{1}, \cdots, L_{r}$ are slowly oscillating functions each of which is either convex or concave from some point on, and if $\beta_{1}, \cdots, \beta_{r}$ are positive numbers less than 1 , then we cannot have (as $n \rightarrow+\infty)$ :

Received by the editors February 8, 1962 .

${ }_{1}^{1}$ This work was supported (in part) by the Office of Naval Research. 


$$
r(n)=n L(n)+\sum_{i=1}^{r} n^{\beta_{i}} L_{i}(n)+o\left(n^{1 / 4} L_{0}(n)\right)
$$

where $L_{0}(n)=(\log n)^{-1 / 2} L(n)^{3 / 4}$ if $L$ is bounded and $L_{0}(n)=(\log n)^{-1 / 2}$ $L(n)^{1 / 4}$ if $L$ is unbounded.

Actually the proof gives the following slightly stronger assertion. If $L, L_{1}, \cdots, L_{r}$ are defined on the interval $[a,+\infty)$ and if we put

$$
v(n)=r(n)-n L(n)-\sum_{i=1}^{r} n^{\beta_{i}} L_{i}(n) \quad(n \geqq a)
$$

and

$$
V(n)=\sum_{a \leqq m \leqq n} v(m)^{2} \quad(n \geqq a),
$$

then it is impossible to have

$$
V(n)=o\left(n^{3 / 2} L_{0}(n)^{2}\right) \quad(n \rightarrow+\infty) .
$$

If a slowly oscillating function $L$ is convex on the interval $[a,+\infty)$, then its second differences for integral values of the variable are non-negative there, that is,

$$
L(n+2)-L(n+1) \geqq L(n+1)-L(n)
$$

for every integer $n \geqq a$. (In fact, for our purposes we could take (5) as the definition of convexity. Cf. [2], pp. 70-72.) From (5) it follows that $L(n+1) \leqq L(n)$ for every integer $n \geqq a$, since otherwise $L$ would change too rapidly to be slowly oscillating. Thus $L(n)$ has a limit, which is either zero or a positive real number.

Similarly, if a slowly oscillating function $L$ is concave on the interval $[a,+\infty)$, we have $L(n+2)-L(n+1) \leqq L(n+1)-L(n)$ for $n \geqq a$. Thus in this case $L(n+1) \geqq L(n)$ for $n \geqq a$ and $L(n)$ has a limit, which is either a positive real number or $+\infty$.

Our theorem concerns the behavior of slowly oscillating functions for integral values of the variable. Their behavior for other values is largely irrelevant as long as continuity and the defining property are preserved.

Throughout $z$ will denote a complex variable inside the unit circle. The proof uses the Erdös-Fuchs method of considering the mean value of $|g(z)|^{2}$ on the circle around the origin with radius $r=e^{-1 / x}$, where $x$ is large.

We shall use the operator $\Delta$ with its usual meaning in the calculus of finite differences. Thus, if $f$ is a function defined on the interval $[a,+\infty)$ and if $n \geqq a$, we have 
$\Delta f(n)=f(n+1)-f(n), \quad \Delta^{2} f(n)=f(n+2)-2 f(n+1)+f(n)$.

\section{Preparatory lemmas.}

Lemma 1. Suppose $b_{0}, b_{1}, \cdots$ are non-negative real numbers such that the series $\sum_{n=0}^{+\infty} b_{n} z^{n}$ converges for $|z|<1$. If $\phi(z)=\sum_{n=0}^{+\infty} b_{n} z^{n}$, if $0<r<1$, and if $0<\alpha<\pi$, then

$$
(2 \alpha)^{-1} \int_{-\alpha}^{\alpha}\left|\phi\left(r e^{i \theta}\right)\right|^{2} d \theta \geqq \frac{1}{3}(2 \pi)^{-1} \int_{-\pi}^{\pi}\left|\phi\left(r e^{i \theta}\right)\right|^{2} d \theta .
$$

Proof. See [1].

LEMMA 2. If $L$ is a slowly oscillating function defined on $[a,+\infty)$ and if $\beta>-1$, then

$$
\sum_{n \geq a} n^{\beta} L(n) e^{-n / x} \sim \Gamma(\beta+1) x^{\beta+1} L(x)
$$

as $x \rightarrow+\infty$.

Proof. Since the sum of the series tends to $+\infty$ as $x \rightarrow+\infty$, we have

$$
\sum_{n \geq a} n^{\beta} L(n) e^{-n / x} \sim \int_{a}^{\infty} u^{\beta} L(u) e^{-u / x} d u \sim x^{\beta+1} L(x) \int_{a / x}^{\infty} v^{\beta} \frac{L(v x)}{L(x)} e^{-v} d v .
$$

In view of (3), the result of the lemma follows from the Lebesgue Dominated Convergence Theorem.

Lemma 3. Suppose $L$ is a slowly oscillating function which is either convex or concave on $[a,+\infty)$. Then if $|z|=e^{-1 / x}$, where $x \geqq a>0$, we have

$$
(1-z)^{2} \sum_{n \geq a} n L(n) z^{n}=O(1)+O(L(x)) .
$$

On the other hand, if $0<\beta<1$, the function $(1-z)^{2} \sum_{n \geq a} n^{\beta} L(n) z^{n}$ is bounded for $|z|<1$.

Proof. Suppose $0<\beta \leqq 1$. Clearly

$$
\begin{aligned}
&(1-z)^{2} \sum_{n \geq a} n^{\beta} L(n) z^{n} \\
&= P(z)+\sum_{n \geq a}\left\{\Delta^{2} n^{\beta} L(n)\right\} z^{n+2} \\
&= P(z)+\sum_{n \geq a}(n+2)^{\beta}\left\{\Delta^{2} L(n)\right\} z^{n+2} \\
&+2 \sum_{n \geq a}\left\{\Delta(n+1)^{\beta}\right\}\{\Delta L(n)\} z^{n+2}+\sum_{n \geq a}\left\{\Delta^{2} n^{\beta}\right\} L(n) z^{n+2},
\end{aligned}
$$


where $P(z)$ is a polynomial in $z$. Let $\lambda=0$ if $L$ is convex and $\lambda=1$ if $L$ is concave. Then $(-1)^{\lambda} \Delta^{2} L(n) \geqq 0$ and $(-1)^{\lambda+1} \Delta L(n) \geqq 0$ for $n \geqq a$. Also we have

$$
0<\Delta n^{\beta} \leqq \beta n^{\beta-1}
$$

and

$$
0 \geqq \Delta^{2} n^{\beta} \geqq \beta(\beta-1) n^{\beta-2} .
$$

Let $M$ be the supremum of $|P(z)|$ for $|z|<1$. Then, putting $r=|z|$ $=e^{-1 / x}$, we have

$$
\begin{aligned}
& \left|(1-z)^{2} \sum_{n \geq a} n^{\beta} L(n) z^{n}\right| \\
\leqq & M+(-1)^{\lambda} \sum_{n \geq a}(n+2)^{\beta}\left\{\Delta^{2} L(n)\right\} r^{n+2} \\
& -(-1)^{\lambda} 2 \sum_{n \geq a}\left\{\Delta(n+1)^{\beta}\right\}\{\Delta L(n)\} r^{n+2}-\sum_{n \geq a}\left\{\Delta^{2} n^{\beta}\right\} L(n) r^{n+2} \\
= & M+(-1)^{\lambda} \sum_{n \geq a}\left\{\Delta^{2} n^{\beta} L(n)\right\} r^{n+2} \\
& -(-1)^{\lambda} 4 \sum_{n \geq a}\left\{\Delta(n+1)^{\beta}\right\}\{\Delta L(n)\} r^{n+2} \\
& -\left\{1+(-1)^{\lambda}\right\} \sum_{n \geq a}\left\{\Delta^{2} n^{\beta}\right\} L(n) r^{n+2} \\
= & M+(-1)^{\lambda} \sum_{n \geq a}\left\{\Delta^{2} n^{\beta} L(n)\right\} r^{n+2} \\
& -(-1)^{\lambda} 4 \sum_{n \geq a} \Delta\left\{\left(\Delta n^{\beta}\right) L(n)\right\} r^{n+2}-\left\{1-3(-1)^{\lambda}\right\} \sum_{n \geq a}\left\{\Delta^{2} n^{\beta}\right\} L(n) r^{n+2} .
\end{aligned}
$$

Now by Lemma 2

$$
\begin{aligned}
\sum_{n \geq a}\left\{\Delta^{2} n^{\beta} L(n)\right\} r^{n+2} & =O(1)+(1-r)^{2} \sum_{n \geq a} n^{\beta} L(n) r^{n} \\
& =O(1)+O\left(x^{-2} \sum_{n \geq a} n^{\beta} L(n) e^{-n / x}\right) \\
& =O(1)+O\left(x^{\beta-1} L(x)\right)
\end{aligned}
$$

and

$$
\begin{aligned}
\sum_{n \geq a} \Delta\left\{\left(\Delta n^{\beta}\right) L(n)\right\} r^{n+2} & =O(1)+r(1-r) \sum_{n \geq a}\left(\Delta n^{\beta}\right) L(n) r^{n} \\
& =O(1)+O\left(x^{-1} \sum_{n \geq a} n^{\beta-1} L(n) e^{-n / x}\right) \\
& =O(1)+O\left(x^{\beta-1} L(x)\right) .
\end{aligned}
$$


Also

$$
\begin{array}{ll}
\sum_{n \geq a}\left\{\Delta^{2} n^{\beta}\right\} L(n) r^{n+2}=O\left(\sum_{n \geqq a} n^{\beta-2} L(n)\right)=O(1) & \text { if } \beta<1, \\
\sum_{n \geq a}\left\{\Delta^{2} n^{\beta}\right\} L(n) r^{n+2}=0 & \text { if } \beta=1 .
\end{array}
$$

Thus the assertions of the lemma follow, since $x^{\beta-1} L(x)$ is bounded if $\beta<1$.

Lemma 4. There is an absolute constant $K$ such that

$$
\int_{-\pi}^{\pi}\left|1-e^{-1 / x} e^{i \theta}\right|^{-1} d \theta<K \log x
$$

for $x>e$.

Proof. Letting $r=e^{-1 / x}$, we have

$$
\begin{aligned}
& \int_{-\pi}^{\pi}\left|1-r e^{i \theta}\right|^{-1} d \theta=\int_{-\pi}^{\pi}\left\{(1-r)^{2}+2 r(1-\cos \theta)\right\}^{-1 / 2} d \theta \\
& =\int_{-\pi}^{\pi}\left\{(1-r)^{2}+r \theta^{2}\right\}^{-1 / 2} d \theta+O(1) \\
& =2 \log (1-r)^{-1}+O(1) \\
& =2 \log x+O(1) \text {. }
\end{aligned}
$$

3. Proof of the theorem. We assume (4) and force a contradiction. In view of (2) and the definition of $v(n)$ we have

$$
\begin{aligned}
g(z)^{2}= & (1-z) \sum_{n \geq a}\left\{n L(n)+\sum_{i=1}^{r} n^{\beta_{i}} L_{i}(n)\right\} z^{n} \\
& +(1-z) \sum_{n<a} r(n) z^{n}+(1-z) \sum_{n \geq a} v(n) z^{n}
\end{aligned}
$$

We get our contradiction by estimating the integral

$$
I(x)=\int_{-\alpha}^{\alpha}\left|g\left(r e^{i \theta}\right)\right|^{2} d \theta \quad\left(r=e^{-1 / x}\right)
$$

both from above and below, for large $x$. Here $\alpha$ will be a function of $x$ to be chosen later, subject to the restrictions $x^{-1}<\alpha<\pi$.

The lower estimate is based on Lemmas 1 and 2. By Lemma 1 and (1) we have 
(7) $\quad I(x) \geqq(3 \pi)^{-1} \alpha \int_{-\pi}^{\pi}\left|g\left(r e^{i \theta}\right)\right|^{2} d \theta=\frac{2}{3} \alpha \sum_{n=0}^{\infty}{c_{n}}^{2} r^{2 n} \geqq \frac{2}{3} \alpha g\left(r^{2}\right)$.

The last step here follows from the fact that, for each $n, c_{n}$ is either 0 or at least 1 and so $c_{n}^{2} \geqq c_{n}$. Let $\gamma$ be a number such that

$$
\max \left(\beta_{1}, \cdots, \beta_{r}, 3 / 4\right)<\gamma<1 \text {. }
$$

Then certainly

$$
|v(n)| \leqq V(n)^{1 / 2}=O\left(n^{\gamma}\right)
$$

and

$$
n^{\beta_{i}} L_{i}(n)=O\left(n^{\gamma}\right) \quad(i=1, \cdots, r) .
$$

In view of Lemma 2, we therefore get from (6)

$$
\begin{array}{rlr}
g\left(r^{2}\right)^{2} & =\left(1-r^{2}\right) \sum_{n \geq a} n L(n) r^{2 n}+O\left(\left(1-r^{2}\right) \sum_{n \geq a} n^{\gamma} r^{2 n}\right) \\
& \sim \frac{1}{2} x L\left(\frac{1}{2} x\right) \sim \frac{1}{2} x L(x) & (x \rightarrow+\infty) .
\end{array}
$$

Thus $g\left(r^{2}\right)>\frac{1}{2} x^{1 / 2} L(x)^{1 / 2}$ for large $x$. Using this in (7), we obtain

$$
I(x)>\frac{1}{3} \alpha x^{1 / 2} L(x)^{1 / 2}
$$

for all sufficiently large $x$.

We now turn to the upper estimate. Using (6) and estimating the integrals of the principal terms on the right side of (6) by means of Lemmas 3 and 4, we obtain for large $x$

$$
I(x) \leqq(A \log x) \max (1, L(x))+\int_{-\alpha}^{\alpha}\left|\left(1-r e^{i \theta}\right) \sum_{n \geq a} v(n) r^{n} e^{i n \theta}\right| d \theta,
$$

where $A$ is a positive number independent of $x$. But by the Schwarz inequality and the trivial inequality

$$
\left|1-r e^{i \theta}\right| \leqq\left|1-e^{i \theta}\right|+\left|e^{i \theta}-r e^{i \theta}\right| \leqq|\theta|+(1-r)<2 \alpha,
$$

we have

$$
\begin{aligned}
\left\{\int_{-\alpha}^{\alpha}\left|\left(1-r e^{i \theta}\right) \sum_{n \geq a} v(n) r^{n} e^{i n \theta}\right| d \theta\right\}^{2} & \leqq 8 \alpha^{3} \int_{-\pi}^{\pi}\left|\sum_{n \geq a} v(n) r^{n} e^{i n \theta}\right|^{2} d \theta \\
& =16 \pi \alpha^{3} \sum_{n \geq a} v(n)^{2} r^{2 n} \\
& =16 \pi \alpha^{3}\left(1-r^{2}\right) \sum_{n \geq a} V(n) r^{2 n}
\end{aligned}
$$


But by our basic assumption (4) and Lemma 2

$$
\begin{aligned}
16 \pi\left(1-r^{2}\right) \sum_{n \geq a} V(n) r^{2 n} & =o\left(\left(1-r^{2}\right) \sum_{n \geq a} n^{3 / 2} L_{0}(n)^{2} r^{2 n}\right) \\
& =o\left(x^{3 / 2} L_{0}(x)^{2}\right) \quad(x \rightarrow+\infty),
\end{aligned}
$$

so that, given a positive number $\delta$, we can say that

$$
16 \pi\left(1-r^{2}\right) \sum_{n \geq a} V(n) r^{2 n}<\delta^{2} x^{3 / 2} L_{0}(x)^{2},
$$

provided $x$ is sufficiently large. Thus we have the estimate

$$
I(x) \leqq(A \log x) \max (1, L(x))+\delta \alpha^{3 / 2} x^{3 / 4} L_{0}(x)
$$

for sufficiently large $x$.

To get a contradiction from the two estimates (8) and (9), we first choose a fixed positive $\delta$ such that

$$
\frac{1}{3} \delta^{-2 / 3}>A+1 \text {. }
$$

For example, we could take $\delta=(3 A+4)^{-3 / 2}$. Then we choose $\alpha$ as a function of $x$ in the following way:

$$
\begin{array}{ll}
\alpha=\delta^{-2 / 3} x^{-1 / 2} L(x)^{-1 / 2} \log x & \text { if } L \text { is bounded, } \\
\alpha=\delta^{-2 / 3} x^{-1 / 2} L(x)^{1 / 2} \log x & \text { if } L \text { is unbounded. }
\end{array}
$$

For large $x$ these choices satisfy the requirement $x^{-1}<\alpha<\pi$. In either case we get a contradiction for sufficiently large $x$. Thus the assumption of (4) is untenable and our result is established.

The Erdös-Fuchs method can also be used to find results for the case where the exponent of the leading term is different from 1 , but it seems likely that the results so obtained are not sufficiently definitive.

\section{REFERENCES}

1. P. Erdös and W. H. J. Fuchs, On a problem of additive number theory, J. London Math. Soc. 31 (1956), 67-73.

2. G. H. Hardy, J. E. Littlewood, and G. Polya, Inequalities, 2nd ed., Cambridge Univ. Press, Cambridge, 1952.

3. J. Korevaar, T. van Aardenne-Ehrenfest, and N. G. de Bruijn, $A$ note on Slowly oscillating functions, Nieuw Arch. Wisk. (2) 23 (1949), 77-86.

UNIVERSITY OF ILLINOIS,

UNIVERSITY OF UTAH, AND

Ohio State University 